\title{
Planting a Poison SEAD: Using Social Engineering Active Defense (SEAD) to Counter Cybercriminals
}

\author{
Matthew Canham ${ }^{1[0000-0001-7638-2023]}$ and Juliet Tuthill2[0000-0002-4640-0005] \\ ${ }^{1}$ Beyond Layer Seven, LLC, Oviedo, FL, USA \\ ${ }^{2}$ University of Central Florida, Orlando, FL, USA \\ mcanhamebelay7. com \\ juliet.tuthill@outlook.com
}

\begin{abstract}
By nearly every metric, the status quo of information security is not working. The interaction matrix of attacker-defender dynamics strongly favors the attacker who only needs to be lucky once. We argue that employing social engineering active defense (SEAD) will be more effective to countering malicious actors than maintaining the traditional passive defensive strategy. The Offensive Countermeasures (OCM) approach to defense advocates for three categories of countermeasures: annoyance, attribution, and attack. Annoyance aims to waste the attacker's time and resources with the objective of not only deterrence but also to increase the probability of detection and attribution. Attribution attempts to identify who is launching the attack. Gathering as much threat intelligence on who the attacker is, provides the best possible defense against future attacks. Finally, attack involves running code on the attacker's system for the purpose of deterrence and attribution. In this work, we advocate for utilizing similar approaches to deny, degrade, and de-anonymize malicious actors by using social engineering tools, tactics, and procedures against the attackers. Rather than fearing the threats posed by synthetic media, cyber defenders should embrace these capabilities by turning these against criminals. Future research should explore ways to implement synthetic media and automated SEAD methods to degrade the capabilities of online malicious actors.
\end{abstract}

Keywords: Social Engineering Active Defense, Offensive Countermeasures, Synthetic Media, Deep Fakes, Cybercrime.

\section{The Security Status Quo is Not Working}

Over the past five years, the FBI's Internet Crime Complaint Center (IC3) logged over two million cybercrime complaints [1]. Only a very small fraction of these complaints will ever result in an arrest, much less a conviction. One of the many reasons these criminals are rarely convicted is that they generally reside outside the legal jurisdictions of the nations in which their victims are located. Another factor to consider is that humans are consistently the primary vectors targeted by cyber criminals [2]. The current state of information security can therefore be simplified as follows; high numbers of cybercrimes are committed, extremely low arrest and conviction rates present little to no deterrence to threat actors, and humans continue to be the attack vector of choice. The traditional models of information protection do not appear to be 
working. Perhaps it is time for the security community to assume a new approach to these rapidly developing problems.

\subsection{Remote Online Social Engineering (ROSE) Attacks}

Social engineering is the primary method of exploiting the human attack surface. Chris Hadnagy defines social engineering as "any act that influences a person to take an action that may, or may not, be in his or her best interests" [3]. Malicious actors use social engineering tactics, techniques, and procedures (TTPs), to manipulate human targets into taking actions that help the attackers achieve their objectives. Attacker TTPs commonly involve impersonating fellow employees, superiors, and persons in need, as a means of emotionally appealing to the target's psychological processes to circumvent critical thinking or evaluation of the attacker's pretext. Attackers simultaneously leverage known psychological phenomena such as social influence principles to manipulate their targets. Such TTPs may include, but are not limited to, inducing stress through threats or time pressure, inducing sympathy through assistance ploys, appeals to ego through aggrandizement, and exploitation of security policy ignorance. The risk-reward ratio for remote online social engineering (ROSE) ploys such as phishing (email-based attacks), vishing (voice-based attacks), and smishing (SMS text-based attacks) is so favorable for cybercriminals that this remains their preferred mode of operation [4]. By launching these attacks from remote locations, often from beyond legal jurisdiction, threat actors assume little risk even if their attack is detected. Technologically mediated communication affords attackers the ability to shape the attack environment and impersonate known associates while exerting a significant degree of perception management against their targets. For example, lateral phishing leverages the saved contact list of a compromised account to launch a "pivot attack" toward a previous contact. By assuming the identity of the compromised account, the attacker shapes the perception of the target to convince them that the phishing email is originating from the trusted contact, thus substantially increasing the chances of a successful attack.

\subsection{Automated Social Engineering}

Automation is a significant ROSE force multiplier because it both increases the attacker's ability to scale and adds capabilities for shaping target perceptions. [5]. Researchers have demonstrated these enhanced capabilities through several projects, examples include the following. The Social Engineering eXposure Index demonstrates the feasibility of automating the reconnaissance stage in social engineering attacks through scripted collection of open-source intelligence [6] [7]. Automated Twitter bots capable of launching spear-phishing attacks demonstrate automated social engineering attack capabilities [8]. Chatbot social engineering has progressed beyond proof-ofconcept as there has been an $820 \%$ increase in chatbot-enabled gift-card scams since the COVID-19 pandemic shift to working from home [9]. The sophistication of these attacks is increasing as recently shown by GPT-3 generation of spear-phishing emails which are more effective than those generated by humans in some cases [10]. 
The emergence, prevalence, and incorporation of synthetic media into ROSE attacks is a recent trend that is causing concern among the security community [11]. Synthetic media is broadly defined as the artificial manipulation, modification, and production of information and includes a wide spectrum of communications media from audio-video deepfakes to text-based chatbots [5]. This new capability massively increases ROSE attacker capability by significantly increasing their ability to impersonate others, thus substantially increasing the number of attacks that they can launch while also elevating pretext believability. Among the growing capabilities and complexities synthetic media presents, deepfake audio and video are perhaps the most concerning. This technology repurposes existing audio or video clips to create digital puppets that can be manipulated through an actor's movements and speech. Using an existing file, a generative adversarial network (GAN) creates a synthetic visual or auditory puppet (synpuppet) that can be manipulated by a human puppet master's movements or speech. This technology has been employed in cinematic entertainment for many years and is used in special effects; however, the recent proliferation of this technology on open-source software repositories means that sophisticated deepfake audio and video are becoming more accessible, therefore deepfake content can be easily generated by threat actors seeking to impersonate unique targets. Recent examples include an attempt to falsify evidence during a custody hearing [12], and multiple uses of deepfake audio to impersonate executives during vishing attacks [13] [14].

\subsection{Is the Best Defense a Good Offense?}

John Strand, the CEO of Black Hills Security, advocates for the employment of Offensive Countermeasures (OCM) in his book by that name [15]. The authors argue that the "old strategies of security have failed us and will continue to fail us unless we start becoming more offensive in our defensive tactics." To these ends, they present three categories of Offensive Countermeasures (OCM): annoyance, attribution, and attack. Annoyance aims to waste the attacker's time and resources with the objective of not only deterrence but also to increase the probability of detection and attribution. The next category, attribution, attempts to identify who is launching the attack. Gathering as much threat intelligence on who is behind the attack provides the best possible defense against future attacks. Attack is the final category of OCM and involves running code on the attacker's system for the purpose of deterrence and attribution. Offensive countermeasures raise many legitimate concerns among skeptics and critics, not the least of which are legality and collateral damage. By "counterhacking" a defender runs the risk of crossing legal boundaries that can put them and their organizations in jeopardy. Considering cybercriminals often utilize third-party proxies to launch their attacks, collateral damage is another serious consideration. The owners of the systems launching attacks are often themselves victims of the attackers, unaware of their systems being compromised. Hacking against these systems with the intention of causing some degree of damage has the potential to inflict harm on innocent third parties. Accounting for this, the authors advocate for poison (a dangerous substance used defensively against predators) rather than venom (a dangerous substance injected into prey by a predator).

The above considerations apply to information technology and the employment technology-based defenses and actions. This poses the question of what degree these 
considerations apply to social engineering (human-focused) attacks. An in-depth discussion of the domestic and international legal implications of offensive countermeasures is beyond the scope of this work; however, we propose that automated and synthetic social engineering TTPs may be used counter-offensively by defenders as a more effective counter to cyber threat actors.

\section{Social Engineering Active Defense (SEAD)}

The emergence of synthetic media has generated a significant level of anxiety within the security community around how these technologies will be used by attackers [5]. Rather than fearing these technologies, defenders should embrace them as additional capabilities to be employed proactively as a defense against ROSE attacks. We propose employing social engineering active defense (SEAD) to deter ROSE attacks, by leveraging synthetic media technology enabled active countermeasures. A SEAD approach leverages one or more of the OCM approaches of annoyance, attribution, and attack to deny, disrupt, and deter cyber threat actors in their efforts. Furthermore, this approach differs from traditional cyber deception in that SEAD deliberately seeks to exploit the cognitive vulnerabilities of attackers for the purposes of annoyance, attribution, and attack.

It should be noted that when employing any variation of active defense, defenders should be mindful of any and all legal regulations and statutes to avoid crossing into criminal territory. This point of caution withstanding, SEAD assumes less potential to cause third-party collateral damage than technologically implemented OCM because SEAD directly engages the attacker.

\subsection{SEAD Annoyance}

If you have ever received an unsolicited phone call from a scammer, you may have wished to impose all manner of ill will upon them. Roger Anderson took this to a new level when he developed the Jolly Roger Bot (JRB). The JRB uses a series of prerecorded voice clips to respond to a scam caller. From the user's perspective, the interaction occurs in the form of an unrecognized call, which the user then redirects to the JRB app. Jolly Roger users have the option of listening to call recordings later to ensure the bots have accurately detected spammers. From the caller's perspective, the app answers the phone appearing to be a human. The bot responds to the sound of the caller's voice with pre-recorded sound clips of random statements. Since the software uses human voice recordings, the responses effectively appear to be human, rather than synthetic. This voice bot has been so effective at wasting scam callers' time that some have remained on the phone conversing with the bot for 20 minutes or more [16]. One recorded interaction captured the caller in conversation with an apparent supervisor, during which the supervisor made the statement "Whenever we get these (voice chatbots)... we have to write the phone number down and then we get them sent to our dialer guy so that we don't get charged for it" [17]. Such outcomes advocate for the effectiveness of this approach in countering voice-based scams. 
As Strand et al (2017) state that annoyance "is about wasting the attacker's time" and resources. Some security-minded individuals have turned this into a sport, known as "scambaiting". Comedian James Veitch has given a series of TED talks about his interactions with email and phone scammers and his efforts to hilariously waste their time [18]. He has been so successful in his efforts that scammers have pleaded with him to stop emailing them [19]. These examples illustrate the potential for SEAD techniques to consume malicious actor resources; however, these countermeasures are being carried out mostly for the entertainment of the researchers. We ask, what might be possible if such OCMs are taken to the next level through AI-driven exploitation of attacker cognitive biases?

Some researchers have proposed employing Oppositional Human Factors as a means of leveraging known design principles against attackers to degrade their efforts [20]. This work explored how cognitive bias influences the perceptions and performance of red team security auditors. Researchers discovered that informing research participants that deception would be used in the study environment not only degraded red teamers' performance but did so in specific and predictable ways. For example, researchers exploited the confirmation bias of red teamers by informing them that a subset of systems were honey pots when in truth all the systems were legitimate. Presented with this information, the red teamers uncovered several false positives, which were systems that they suspected to be illegitimate. The participants even went to great lengths in collecting evidence that these resources were false, thus diverting their attention away from legitimate targets [20]. Covert Impairments have also been proposed as intentional mechanisms to deter and degrade attackers through the employment of subtle forcing functions to "guide" attacker actions in directions that are advantageous for the defenders [21].

We propose that leveraging existent technologies such as the JRB, combined with covert impairments that deliberately target the cognitive biases of attackers, could be a highly effective deterrent against human hackers that use social engineering tactics to compromise their targets. Such an approach would also present a likely counter to automated attacks in a manner demonstrated by a video depicting two chatbots in a conversation [22]. Furthermore, if such techniques are employed strategically, they could provide valuable threat intelligence about the attackers.

\subsection{SEAD Attribution}

Several researchers have proposed that honey pots could be repurposed to infer personality traits and other individual characteristics of attackers [23]. Employing a series of forced actions, similar in nature to those techniques used by magicians, defenders may be able to create an illusion of choice for the attackers that causes them to reveal individually attributable characteristics [24]. Sometimes referred to as 'inference attacks', behavioral actions [25], or cognitive preferences [26] can reveal characteristics of an unidentified individual. Malin (2021) argues that Locard's Exchange Principle applies to digital artifacts and that every interaction within and across digital space will necessarily leave behind digital trace evidence which can be later analyzed. Aggregated, these digital traces give way to the Watanabe Principle of Digital Artifact Exchange which posits that these can be leveraged to derive attribution to a particular offender [27] [28]. If such exchanges can be deliberately manipulated or 
induced, it may be possible to create 'Cognitive Fingerprints' through a series of 'covert games', as some suggest [26]. Wixey refers to this process as finding the person behind the machine [29]. While there is still much work to be done within this domain, these SEAD tactics focus on actions taken within the defender's perimeter, a more aggressive posture might carry the fight beyond friendly territory by taking actions against criminal-controlled assets.

\subsection{SEAD Attack}

An excellent example of a successful SEAD attack is the Honey Phish Project. In this project, researcher Robbie Gallagher, set up several fake email addresses to act as mailbox honey pots for phishing scammers. These Honey Phish would automatically reply to phishing emails with an email containing a uniquely generated link, that when clicked, generated client-side browser fingerprinting, sending identifiable information back to the researcher [30]. Another example can be found in the scambaiting community with a researcher going by the handle Engineer Man, who used a Python bot to insert false usernames and passwords into a malicious website to the point of filling up the scammer's database [31].

Gift-card scams have increased substantially since the remote working trend due to the COVID pandemic. These scams often follow a similar script of the criminal employing a pretext to convince the victim to purchase gift cards, scratch off the coating obscuring the redemption code on the back and sending an image of this code to the scammer, who uses this code to obtain the funds on the card. A potential SEAD attack to counter these gift card scams might include embedding a tracking pixel that relays attributable information about the attacker's device, location, and possibly their identity back to defenders. One reason these examples are likely to enjoy a high level of success is that the attackers do not expect that they themselves will become victims of an attack. We again strongly advise any researchers exploring these lines of SEAD to consult with legal experts before conducting this type of research.

\section{$3 \quad$ Future Directions, Research Questions and Challenges}

We posit that active defense against malicious social engineering attacks will be more effective in countering malicious attacks than the status quo approach of continuing to rely on passive defense strategies. To shift the balance of power back in favor of the defenders, future research into SEAD should pursue the following research avenues.

\subsection{Identifying an Attack}

Among the more formidable challenges to implementing automated SEAD approach will be detecting that a malicious social engineering attack is occurring, as opposed to a benign interaction. Indeed, the overarching challenge of countering social engineering is that successful attacks are frequently not recognized as malicious attacks. This makes automating the identification process extremely challenging. Some researchers have identified a potential bottleneck that all social engineering attacks must pass through, by either asking for private information or directing the target to take an adverse action 
[32]. Understanding that this conversational bottleneck exists allows automated natural language processing (NLP) algorithms to constrain focus on conversational features within this scope. Other researchers have explored incorporating NLP with other algorithms to detect grammar anomalies and check for the presence of social influence principles [33]. Another approach may be to create digital cognitive clones of human operators to emulate human responses to social engineering attempts [34]. A significant amount of work remains to be explored within this field.

\subsection{Annoyance}

Once a potential attack is identified, the question of how to best degrade attacker capabilities comes in focus. Future research in this area should ask to what extent can automated SEAD bots waste the time and resources of attackers through social engineering and covert impairments? What factors will most degrade attacker capabilities? To what extent can voice-bots deceive attackers into believing that the bots are humans? Which characteristics of the voice-bots most contribute to this deception? In this effort, more investigative efforts should explore ways in which Cialdini's principles of influence and magnetizers (mysterious, self-relevant, and unfinished) may enhance the efficacy of SEAD annoyance efforts [35]. A recent study on phishing susceptibility found that simulated phishing emails which included a greater number of these magnetizer techniques resulted in substantially higher clickrates than did emails employing fewer magnetizers [36]. We suggest that these same techniques might be employed against attackers to consume more of their attention, time, and resources, and potentially lead them to reveal attributable characteristics about themselves.

\subsection{Attribution}

Previous work in human side-channel attacks and forensic linguistics reveals that significant insights may be derived from data that appears to be innocuous [4]. Future work should seek to maximize attributable inferences and identify which types of data are most likely to reveal crucial insights. A few questions that researchers might explore include the following. Which attacker attributes are most likely to be uncovered by various SEAD measures? To what extent can the attacker's psychological characteristics and cognitive biases be used against them to reveal critical threat intelligence? Which TTPs will most effectively allow defenders to cognitively fingerprint attackers? Which forms of digital trace evidence are most likely to be uncovered by SEAD bots? Beyond identifying who potential attackers are, it might be possible to reveal more about them by utilizing TTPs that go "beyond the perimeter" of defenders' systems.

\subsection{Attack}

Future work on SEAD attacks could potentially move in two parallel tracks, one which focuses on improving technological capabilities, and another which focuses on legal and regulatory questions. Improving technical countermeasures that will interfere with 
criminal activities without causing collateral damage to innocent third parties should be the priority for technology focused research. Legal research should clarify how existing domestic and international legal frameworks might better accommodate offensive countermeasures, or alternatively how legislation can be passed to better accommodate SEAD actions that extend into criminal systems to deter future malicious actions and to assist law enforcement. Questions to be answered include, to what extent will SEAD methodologies fall within the scope of computer abuse regulations? How can SEAD TTPs stay within the boundaries of being legal while still presenting an effective counter to malicious cyber actors?

The status quo of information security appears to be failing. It is time for the security community to adopt new approaches. Social Engineering Active Defense (SEAD) represents such an approach. Exploring these lines of research will allow the security community to proactively mitigate malicious attacks and potentially tilt the balance of power in the defenders' favor.

\section{$4 \quad$ References}

1. FBI, Internet Crime Complaint Center (IC3). Internet Crime Report 2020. Washington, DC, USA. (2021b).

2. Verizon. DBIR 2021 Data Breach Investigations Report. Verizon, New York, NY, USA. (2021).

3. Hadnagy, C. Social Engineering: The Science of Human Hacking. John Wiley \& Sons. New York, NY, USA. (2018).

4. Wixey, M. Every ROSE Has its Thorn: The Dark Art of Remote Online Social Engineering Black Hat USA, Las Vegas, NV, USA. (2018).

5. Canham, M. Deepfake Social Engineering: Creating a Framework for Synthetic Media Social Engineering. Black Hat USA, Las Vegas, NV, USA. (2021).

6. Wilkerson, W. S., Levy, Y., Kiper, J. R., \& Snyder, M. Towards a development of a Social Engineering eXposure Index (SEXI) Using Publicly Available Personal Information. 2017 KSU Conference on Cybersecurity Education, Research, and Practice. Kennesaw State University, Kennesaw, GA, USA. (2017).

7. Wilkerson, W. S. Development of a Social Engineering eXposure Index (SEXI) Using Open-Source Personal Information. Doctoral Dissertation, Nova Southeastern University, Fort Lauderdale-Davie, FL, USA. (2021).

8. Seymour, J., \& Tully, P. Generative models for spear phishing posts on social media. arXiv preprint arXiv:1802.05196. (2018).

9. $820 \%$ Jump In E-Gift Card Bot Attacks Since COVID-19 Lockdowns Began, https://www.techrepublic.com/article/820-jump-in-e-gift-card-bot-attacks-since- covid-19lockdownsbegan/, last accessed 2021/03/21.

10. Lim, E., Tan, G., Hock, T., \& Lee, T. Turing in a Box: Applying Artificial Intelligence as a Service to Targeted Phishing and Defending Against AI-Generated Attacks. Black Hat USA, Las Vegas, NV, USA. (2021).

11. FBI. Malicious Actors Almost Certainly Will Leverage Synthetic Content for Cyber and Foreign Influence Operations. Federal Bureau of Investigations Private Industry Notification. Washington, DC, USA. (2021a).

12. 'Deepfake' Audio Evidence Used in UK Court to Discredit Dubai Dad, https://www.thenationalnews.com/uae/courts/deepfake-audio-evidence-used- in-uk-courtto-discredit-dubai-dad-1.975764, 2021/10/26. 
13. Fraudsters Used AI to Mimic CEO's Voice in Unusual Cybercrime Case, https://www.wsj.com/articles/fraudsters-use-ai-to-mimic-ceos-voice-in-unusualcybercrime-case-11567157402, last accessed 2020/07/29.

14. Fraudsters Cloned Company Director's Voice in $\$ 35$ Million Bank Heist, Police Find., https://www.forbes.com/sites/thomasbrewster/2021/10/14/huge-bank-fraud-uses-deepfake-voice-tech-to-steal-millions/?sh=35955ba77559, last accessed 2021/10/14.

15. Strand, J., Asadoorian, P., Robish, E., Donnelly, B. \& Galbraith, B. Offensive Countermeasures: The Art of Active Defense. CreateSpace Independent Publishing Platform, Scotts Valley, CA, USA. (2017).

16. Telephone Spam/Scam Problem? Bring In the Robots, https://www.youtube.com/watch?v=UXVJ4JQ3SUw, last accessed 2022/01/02.

17. Absolute Proof that Jolly Roger Telephone is Disrupting the Vacation Cruise Telemarketers, https://www.youtube.com/watch?v=ezZ2V1CH32E, last accessed 2022/01/02.

18. This Is What Happens When You Reply to Spam Email. TED Global Geneva, https://www.ted.com/talks/james_veitch_this_is_what_happens_when_you_reply_to_spa m_email?language $=$ sc\#t-149006, last accessed 2021/12/07.

19. Ultimate troll, https://www.youtube.com/watch?v=3MHDDSekvcE, last accessed 2021/12/22.

20. Gutzwiller, R., Ferguson-Walter, K., Fugate, S., \& Rogers, A. "Oh, Look, A Butterfly!” A Framework for Distracting Attackers to Improve Cyber Defense. In Proceedings of the Human Factors and Ergonomics Society Annual Meeting (Vol. 62, No. 1, pp. 272-276). Sage Publications, Los Angeles, CA, USA. (2018).

21. Monaco, J., V. "Bug or Feature? Covert Impairments to Human Computer Interaction." In Proceedings of the 2020 CHI Conference on Human Factors in Computing Systems, pp. 115. 2020.

22. AI vs. AI. Two Chatbots Talking To Each Other, https://www.youtube.com/watch?v=WnzlbyTZsQY,last accessed 2021/12/22.

23. Odemis, M., Yucel, C., Koltuksuz, A., \& Ozbilgin, G. Suggesting a Honeypot Design to Capture Hacker Psychology, Personality and Sophistication. In 13th International Conference on Cyber Warfare and Security. Academic Conferences, Washington, DC, USA. (2018).

24. Macknik, S., Martinez-Conde, S., \& Blakeslee, S. Sleights of Mind: What The Neuroscience Of Magic Reveals About Our Everyday Deceptions. Henry Holt and Company. New York, NY, USA. (2010).

25. Naini, F. M., Unnikrishnan, J., Thiran, P., \& Vetterli, M. Where You Are Is Who You Are: User Identification by Matching Statistics. IEEE Transactions on Information Forensics and Security, 11(2), 358- 372. IEEE, New York, NY, USA. (2015).

26. Abramson, M. Cognitive Fingerprints. In: 2015 AAAI Spring Symposium Series. Palo Alto, CA, USA. (2015).

27. Watanabe, D. Digital Behavioral Criminalistics: The Art and Science. In Proceedings of the 9th Annual Mid-Atlantic INLETS: Violent Crimes and Terrorism Trends, Washington, DC. (2018).

28. Malin, C. Digital Behavioral Criminalistics to Elucidate the Cyber Pathway to Intended Violence. In: Meloy, J. R., Hoffmann, J. (eds.) International Handbook of Threat Assessment (2nd Ed). Oxford University Press, Oxford, UK. (2021).

29. Wixey, M. Betrayed by the Keyboard: How What You Type Can Give You Away

30. Where Do the Phishers Live? Collecting Phishers' Geographic Locations from Automated Honeypots. ShmooCon 2016, https://shmoo.gitbook.io/2016-shmooconproceedings/one_track_mind/06_where_do_the_phishers_live, last accessed 2021/12/07.

31. Showing A Craigslist Scammer Who's Boss Using Python, https://www.youtube.com/watch?v=UtNYzv8gLbs, last accessed 2021/12/07. 
32. Sawa, Y., Bhakta, R., Harris, I. G., \& Hadnagy, C. Detection of Social Engineering Attacks Through Natural Language Processing Of Conversations. In 2016 IEEE Tenth International Conference on Semantic Computing (ICSC) (pp. 262-265). IEEE, New York, NY, USA. (2016).

33. Lansley, M., Mouton, F., Kapetanakis, S., \& Polatidis, N. SEADer++: Social Engineering Attack Detection in Online Environments Using Machine Learning. Journal of Information and Telecommunication, 4(3), 346-362. Taylor \& Francis, London, UK. (2020).

34. Golovianko, M., Gryshko, S., Terziyan, V., \& Tuunanen, T. Towards Digital Cognitive Clones for The Decision-Makers: Adversarial Training Experiments. Procedia Computer Science, 180, 180-189. Elsevier: Amsterdam, The Netherlands. (2021).

35. Cialdini, R. Pre-Suasion: A Revolutionary Way to Influence and Persuade. Simon and Schuster. New York, NY, USA. (2016).

36. Canham, M., Dawkins, S., \& Jacobs, J. Manuscript Under Review. 